\title{
22nd Annual Miami Breast Cancer Conference*
}

Almost 1000 delegates from 25 countries gathered for the 22nd Annual Miami Breast Cancer Conference. Dr. Dan Osman, the Founder and Director of the meeting, made it one of the prime breast cancer meetings worldwide. The conference is co-chaired by Dr. DerHagopian and Dr. Love, both from Miami, FL.

The plenary session on Thursday morning started off with a series of lectures followed by keypad questions; the lectures were ranging from 'exploiting the hallmarks of breast cancer' up to 'management of benign, premalignant and rare breast cancers'. The modern knowledge of molecular pathogenesis and identification of targets was at the center of this session that included identification of tumor subtypes which can be used as prognostic factors but additionally, as predictive markers for specifically targeted therapy. In particular, the following targets were discussed: estrogen receptor (Anastrozole, Tamoxifen, Alone and in Combination (ATAC) study update by Dr. Howel); human epidermal growth factor receptor (HER) 2 receptor as synergism or resistance to treatment, and role of HER3 and HER4 (Dr. Pegram); concept of dual receptor inhibition, using epidermal growth factor receptor (EGFR) and HER2 as targets (Dr. Burris); signal transduction down to the regulation and inhibition of the cell cycle (Dr. Dowsett); apoptosis as a target (Dr. Tripathy) and angiogenesis (Dr. Miller). It is quite clear that modern molecular approach will be soon an integral part of therapy for breast cancer. Once this approach reaches earlier stage of breast cancer (as did chemotherapy and endocrine intervention), in the future it will certainly contribute to improved clinical outcomes.

Benign breast disease and its management was discussed in the second part. A very lively case discussion took part with the scientific panel and the audience.

During lunch break, a number of different small group lunches were conducted and led to a real intensive interaction between participants.

\footnotetext{
*Twenty-second Miami Breast Cancer Conference, Loews Miami Beach Hotel, South Beach, Miami, Florida, USA, 23-26 February 2005

Correspondence to: Stefan Glück, Professor of Medicine, Miller School of Medicine, University of Miami; Clinical Director, UMSylvester Comprehensive Cancer Center, Braman Family Breast Cancer Institute, 1475 NW 12th Avenue, Miami, FL 33136, USA. E-mail: sgluck@med.miami.edu; Tel: +1 305243 4909; Fax: +1 3052434975

Publication date 29/04/05 BCO/451/2005/CR
}

In the afternoon, an extensive session on the modern findings, management including surgical and radiation of ductal carcinoma in situ (DCIS) was discussed, and followed by another panel discussion. At the same time, systemic therapy of breast cancer and its novel approaches were presented. Dose scheduling of chemotherapy was mentioned, and clearly it might be one of the options to improve treatment delivery.

Aromatase inhibitors have come a long way since they were discovered more than 30 years ago; today it is the question of how and when to use them: we do have now solid data that they can replace tamoxifen with the goal to reduce the number of recurrences, contralateral breast cancer with less side effects. It is only a matter of time until we will be able to show even survival benefit of this class of compounds over tamoxifen. After these sessions, 'meet the professor' sessions in surgery and medical oncology concluded the formal program.

Prior to the 'get acquainted reception' in the early evening, a satellite symposium 'From Principle to Practice', chaired by Dr. Glück from Miami, focused on the use of anthracyclins, taxanes and the delivery of these drugs. Dose density and dose intensity were the focus of this comprehensive overview of how and when to use adjuvant chemotherapy. It is clear today that modern anthracyclins and taxanes are part of the most effective adjuvant treatments that led to a decrease of mortality over the past 18 years or so. This all happens in spite of the fact that in North America alone, we have been observing increasing incidence of breast cancer, up to 7000 cases per year more than in previous years. Adjuvant chemotherapy certainly is one of the treatment modalities that improve outcomes of breast cancer.

Friday morning was dedicated to invasive carcinoma of the breast: surgical management of suspicious lesions, radio guided localization, imaging, pathology reporting and management after preoperative systemic treatment were the most topics discussed. Very interesting and controversial questions around long-term follow-up of BRCA1/2 patients were another important contribution on Wednesday morning. Dr. Mel Silverstein was the recipient of the 2005 Miami Breast Cancer Award for his outstanding contribution to patients with breast cancer. The tumor board panels have been designated to address non-protocol use of adjuvant and neo-adjuvant 
(preoperative) systemic therapy. The role of taxanes (docetaxel and paclitaxel) and anthracyclins (doxorubicin and epirubicin), and their appropriate dosing and scheduling were discussed. The addition of trastuzumab and other novel compounds for the management of patients with metastatic disease was also discussed in an interactive manner with the audience.

Friday afternoon, two breakout sessions were scheduled independently: surgical and onco-plastic surgery, and medical oncology; both followed by 'meet the professor' sessions with corresponding specialists.

In the evening, a wonderful get together reception and party took place outside in the wonderful 'backyard' of the hotel, at poolside. Weather was great, food and open bar helped to please, and live music entertained participants and their companions.

Saturday morning was the last half-day of the meeting and delegates were discussing management of locoregional recurrences, adjuvant therapy including endocrine intervention and issues of premenopausal women who wish to preserve fertility.
Focus on preoperative chemotherapy concluded this very focused and informative meeting where questions for day-to-day problem solving as well as innovative and experiential treatment of patients with breast health problems were extensively addressed.

During scientific breaks, South Beach offered a lot for all participants at the sandy beach with turquoise ocean as did the night with a wide selection of restaurants, lounges and night clubs. This conference is now well established within the community of health care professionals who deal with breast cancer and will be again well attended next year during the month of February. Certainly, the vibrant city of Miami Beach and the weather in South Florida adds to the incentive to participate.

$$
\begin{array}{r}
\text { Stefan Glück, MD, PhD } \\
\text { Clinical Director } \\
\text { UMSylvester Comprehensive Cancer Center } \\
\text { Braman Family Breast Cancer Institute } \\
\text { Miami, FL, USA }
\end{array}
$$

